\title{
Void Aware Position Based Opportunistic Routing for QoS in Mobile Ad Hoc Networks
}

\author{
V. Raji', N. Mohan Kumar ${ }^{2}$ \\ ${ }^{1}$ Department of Computer Science and Engineering, S.K.P. Engineering College, Thiruvannamalai, India \\ ${ }^{2}$ Department of Electronics and Communication Engineering, S.K.P. Engineering College, \\ Thiruvannamalai, India \\ Email: raji.vpm@gmail.com,nmkphdju@gmail.com
}

Received 8 April 2016; accepted 20 May 2016; published 15 June 2016

Copyright (C) 2016 by authors and Scientific Research Publishing Inc.

This work is licensed under the Creative Commons Attribution International License (CC BY).

http://creativecommons.org/licenses/by/4.0/

(c) (i) Open Access

\section{Abstract}

The unique anywhere, anytime wireless communication support offers, tremendous potential for the next generation of applications in a Mobile Ad-hoc Network (MANET). The Quality of Service (QoS) has been the ever demanding task of wireless communication to satisfy the application requirements. Geographical routing employs a greedy forwarding technique to deliver the packets to the destination and to owe the communication void, it fails to render the expected level of QoS. Opportunistic routing technique effectively utilizes the advantages of broadcasting nature of the wireless medium and selects a set of forwarding candidates instead of relying on a greedy node. To improve the efficiency of QoS routing in sparse and highly dynamic network topology, this paper proposes the Void-Aware Position based Opportunistic Routing (VAPOR). The VAPOR maintains 2-hop neighbor information to take a routing decision, but it is limited to 1-hop information when the node density is high. It efficiently balances the storage overhead and communication delay due to void and it increases the network throughput even under a sparse network. To provide a certain assurance level for packet reachability, VAPOR decides the potential forwarders based on the forwarding probability that measures link stability, capacity, and connectivity factor. It adaptively favors a path that avoids frequent link failure and unreliable link usage. By limiting the propagation area of duplicate packets, VAPOR reduces wastage of network resources, and it takes the advantage of concurrent batch forwarding to avoid further duplication and unnecessary delay.

\section{Keywords}

Mobile Ad Hoc Network, Opportunistic Forwarding, QoS Routing, Void Alleviation 


\section{Introduction}

In a MANET, the mobile nodes form a self-organized network in a shared wireless medium without the aid of permanent infrastructure. Each node in the wireless network has limited transmission range. Therefore, multiple nodes are employed in provisioning end-to-end communication. Unlike the topology based routing, the geographic routing utilizes the physical location information to provide efficient hop-by-hop routing [1]. In multi-hop wireless communication, the unpredictable node mobility and frequent changes in the node connectivity lead to a communication hole in the network. If there is no forwarding node in the coverage area of the sender node, the greedy routing fails, resulting in degradation of performance [2]. An important research issue in QoS routing is to meet both the throughput and the reliability of wireless communication. Providing a reliable and high throughput routing is a challenging problem due to the varying network topology and traffic conditions. Under such conditions, the greedy routing may lead to multiple transmissions to deliver a packet to the next hop. The opportunistic routing has been introduced to provide the assurance for packet reachability. It employs the broadcasting nature of the communication medium that leads to the multiple receptions of data packets within the communications range. Therefore, multiple potential neighbors get the opportunity to forward the data to combat with unreliable wireless links [3]. The opportunistic routing provides efficient wireless communication and avoids failure using multiple forwarding candidates. Among the one-hop neighbors, it selects the next forwarder of the packet based on its proximity to the destination and it suppresses the lower priority forwarding candidates.

A greedy selection is not an optimal solution for deciding the forwarding candidates to support QoS [4]. The QoS in opportunistic routing faces major challenges due to unpredictable node mobility, the communication hole and duplicate transmissions in the network. The routing decision based on 1-hop neighbor information performs better in the dense network, whereas an effective mechanism is necessary to handle the communication hole in sparse networks. Prioritization of multiple receivers based on its proximity to the destination is not an optimal solution in a highly mobile scenario. Moreover, the improper coordination among multiple packet receivers results in duplicate transmissions. The existence of a communication hole in the network is a critical issue, and traditional void handling methods fail to tackle the routing issues on the void [5]. Thus, it is essential to propose a multi-objective protocol to deliver the QoS in terms of both throughput and reliability irrespective of node density exploiting the robustness of opportunistic forwarding.

VAPOR improves the routing performance of highly dynamic and sparse network topology and delivers both the throughput and the reliability. The proposed VAPOR supports the QoS routing and enhances the efficiency of packet forwarding by exploiting the broadcast nature of the wireless medium. It employs the 1-hop routing information to take a routing decision, but in sparse network topology, it determines the communication hole in advance by considering the local map for 2-hop information. The VAPOR broadcasts the data packets to all the forwarding candidates in a single transmission using Media Access Control (MAC) interception. Furthermore, it prioritizes the packet receivers according to its forwarding probability, which has two metrics such as the forwarding score and the connectivity factor to improve the network throughput. It elects a high probability forwarding candidate as the best forwarder of the packet, and other forwarding candidates await at the end of the batch to receive all the data packets. The batch map marks all the packets transmitted by the best forwarder and attaches this information to each data packet [3]. The packets unmarked in the batch map are transmitted by the next priority candidates in order, concurrently the subsequent batch of packets is transmitted through the best forwarder selected from the current 2-hop forwarding set. Thus, it increases the reliability and the throughput. Finally, the performance evaluation shows that the proposed VAPOR protocol provides efficient QoS routing.

\subsection{The Main Contributions}

The proposed work, VAPOR improves QoS of opportunistic routing in both the throughput and the reliability, by handling two extreme scenarios such as high mobility and sparse network topology.

In VAPOR, the link stability estimation service provided by the lower layer increases the efficiency of the void prediction mechanism. In this approach, each node predicts when void occurs within a two-hop forwarding region due to node mobility.

The proposed VAPOR increases the packet forwarding efficiency significantly by allowing the best forwarder to relay the packet first and the forwarding candidate takes a turn to relay the packet in order, when it believes that certain packets fail to transmit. 
VAPOR follows the batch operated the transmission in which the packet forwarder shows a clear view of the transmitted packet information to the remaining forwarders using batch map for reducing duplication and wastage of network resources. It exploits the advantage of concurrent transmission to reduce the additional delay of batch operated transmission.

The restriction of forwarding region and conversion of probability values into real integer values between 0 and 10 minimizes the required memory space and storage overhead. The matrix storage system decreases the data retrieval time that intuitively supports the opportunistic routing to progress without additional delay.

\subsection{Paper Organization}

The paper is organized as follows: The section 2 discusses the previous works related to the opportunistic routing. The proposed VAPOR routing scheme is discussed in section 3. Section 4 shows the experimental results of the proposed VAPOR routing and Section 5 concludes the work.

\section{Related Works}

This section illustrates the existing opportunistic routing protocols, and the works that are related to the current QoS routing, the void handling techniques, the bandwidth, and the stability estimation. The impact of insufficient routing QoS metrics due to the network constraints on wireless communication are surveyed in [6]. In addition, these works illustrate the existing solutions to the problems above to attain the required routing QoS for wireless communication. To increase the reliability of wireless communication, the multipath routing has been proposed [7]. In the dynamic network topology, all the paths may be disconnected with high probability due to unpredictable node mobility. Moreover, the multipath routing is incapable to provide the uninterrupted data delivery when the path length is high. The impact of unpredictable node mobility on the long path is higher than the short path. Recently, to improve the efficiency of packet forwarding, broadcasting nature of the wireless medium has been exploited in opportunistic routing that significantly improves the communication throughput and reliability [3]. A large number of opportunistic routing protocols have been proposed [8]-[11]. The position based routing, named as opportunistic geographic routing assigns the forwarding candidates based on packet progress in each hop. In [12], the opportunistic re-transmission protocol is proposed to combat with the unreliable wireless connections. Multicast-like routing technique [13] also exploits the broadcasting nature of the wireless medium, but it selects and prioritizes the forwarding candidates based on its proximity to the destination. However, none of the existing works utilizes the advantage of greedy routing, but it is not an optimal solution for QoS provisioning. Many protocols have been proposed in the unicast routing with QoS provisioning [14] [15]. It improves two domains such as timeliness and reliability to support the QoS. It exploits inherent redundancy in the reliability domain for releasing multipath, and achieving service differentiation. An efficient location prediction mechanism [16] works together with geographical routing for attaining the QoS.

Several void handling techniques have been proposed in MANET [5] such as Greedy Perimeter Stateless Routing (GPSR) [17], stateless QoS [18], and Greedy-Face-Greedy (GFG) [19]. In these protocols, the routing process is started with the greedy mode, and it is switched over to the recovery mode when the communication hole is created. For every change of the local topology, each node ensures whether the planar graph connectivity is altered and whether any vertex is added or removed from the graph. It induces high routing overhead in the network [20] [21]. Another solution for void handling is the virtual based schemes. This scheme elects the trigger node to carry the data packets towards the virtual destination. However, it induces duplicate forwarding and causes additional communication delay in the data transmission.

\section{Basic Idea of Proposed VAPOR Protocol}

The proposed multi-objective opportunistic routing alleviates the communication hole, by determining 2-hop forwarding set to meet both throughput and reliability constraints. For both sparse and dense network, VAPOR estimates 2-hop forwarding set. But, it applies different methods in providing forwarding priority to 1-hop neighbors. The advantage of 2-hop forwarding scheme is that it overcomes the weakness of communication hole in MANET. In other words, to handle the communication hole in the network, the proposed VAPOR employs two rounds of beacon broadcasting to identify the void nodes in advance. It forms 2-hop forwarding set that excludes void nodes, and it avoids unnecessary communication delay on void handling. In [5], it states that the prediction of the communication-hole is not feasible in a network with unpredictable dynamic changes. 
Unlike existing void handling techniques, VAPOR predicts the void nodes accurately with the support of link stability estimation scheme provided by the physical layer. Prioritizing the nodes in a 2-hop forwarding set according to the forwarding probability is based on per hop link stability, capacity, and connectivity factor. For high-density networks, the VAPOR takes into account 1-hop neighborhood information, but it changes its routing mode to 2-hop forwarding set in sparse networks for estimating the forwarding probability. It is because the opportunity for reliable routing is less in a sparse network. It achieves a balance between reliable routing and overhead and enhances the QoS routing in terms of throughput and reliability in both sparse and dense network.

The higher priority node is set as the best forwarder and others as forwarding candidates. In VAPOR, the best forwarder's transmission suppresses the lower priority forwarding candidates [10]. The forwarding candidates relay the packets when it is not forwarded by the best forwarder. Due to the failure of overhearing the packet transmitted by the best forwarder, the forwarding candidate accumulates duplicate transmissions. When the best forwarder fails to transmit, the forwarding candidate follows the unicast routing instead of multicast-like routing. It selects a node in the forwarding list of the best forwarder, such that forwarding candidate's transmission ensures the nodes in the forwarding region of the best forwarder that can overhear and discard the duplicate packets successfully.

To avoid duplicate transmission, a source node describes a set of data packets as a batch and follows scheduling transmissions [3]. Each node caches the received packets in a packet list for a particular time slot. A node in the forwarding region easily identifies the duplicate transmissions by ensuring the packet list. In case the best forwarder fails to transmit the packet, the forwarding candidate selects a node from the forwarding list of the best forwarder and transmits the packet as unicast. If it is already received, the receiving node drops the duplicate packet, and informs the forwarding candidate. Thus, the duplicate packet transmission ends within a 1-hop, thereby achieving multi-objective QoS in the domain of on-time-reachability and reliability.

\subsection{System Model}

Consider a wireless mobile ad-hoc network with an area Height $(H)$ and Width $(W)$ as a graph $G(V, C) . V$ represents a set of mobile nodes in the network and $|V|=N$. $C$ represents the set of direct connections between the mobile nodes. Each node is directly connected with others that are placed within the radio range $(\Re)$ and $N \in \mathfrak{R}_{s}$ called as 1-hop neighbor set Ns. The direct connection denotes that the forwarding candidate. The set of Ns is limited to a particular angle $(\alpha)$ between the source and the destination $\angle \alpha \Re_{s}$ are defined as one hop forwarding set $\left\{S_{N}\right\}$ and it has 2-hop forwarding set $\left\{S_{2 N}\right\}$, which also contains the nodes lying in a particular angular range toward destination. Ns is located within $\Re$. All nodes $\in\left\{S_{N}\right\}$ participate in the opportunistic local forwarding.

$$
\begin{gathered}
N s=\{V\} \in \mathfrak{R}_{s} \\
S_{N}=\left(S, N_{s}\right) \in C \wedge\left(N_{s} \in \angle \alpha\left(\mathfrak{R}_{s}\right) \& N_{2 S}\left(\forall N_{s}\right) \neq 0\right) \quad \text { where } i=1,2, \cdots,\left|\angle \alpha\left(N \in \mathfrak{R}_{s}\right)\right| \\
S_{2 N}=\left(S_{N}, N_{2 S}\right) \in C \wedge\left(N_{2 S} \in \angle \alpha\left(\mathfrak{R}_{F}\right) \& V\right) \quad \text { where } i=1,2, \cdots,\left|\angle \alpha\left(N \in \mathfrak{R}_{s}\right)\right| .
\end{gathered}
$$

The node density or $|N s|$ divides the network $G$ into Sparse $\left(G_{s}\right)$ and Dense network $\left(G_{d}\right)$. Node density can influence the behavior of an opportunistic routing system. The $G_{d}$ network with high $|N s|$ results in better reliability, but when the number of nodes in $N s$ is low or in $G_{s}$, the QoS routing tends to be poor. To improve the QoS routing, VAPOR takes both $\left\{S_{N}\right\}$ and $\left\{S_{2 N}\right\}$ into account to prioritize nodes according to the metrics provided by multiple layers in $G_{s}$, whereas in $G_{d}$ it is sufficient to consider only $\left\{S_{N}\right\}$ in prioritizing the nodes. For example, PHY layer provides link stability in terms of Received Packet Signal Strength (RPSS) and MAC layer provides capacity estimation service $\forall C \in S_{N}$. MAC layer employs IEEE 802.11e for ensuring the QoS. F1 with a higher priority has high stability and capacity than $\left\{S_{N}-F 1\right\}$ and moreover, $\left\{S_{N}-F 1\right\}$ always expects $F 1$ to relay the packet first. If $F 1$ fails to transmit the packet, the next priority node F11 takes a turn to relay the packet to one of the node $\in\left\{S_{N}\right\}$ of $F 1$.

\subsection{2-Hop Forwarding Set for Void Alleviation}

The VAPOR introduces 2-hop forwarding set for void alleviation to improve the network throughput. In VAPOR, it assumes that every node is aware of its own and the destination location information using GPS re- 
ceiver. Every node retains the local map for 2-hop information through the exchange of location information among 2-hop neighbors. It means that each node gathers the neighbor list as well as 1-hop neighbors of each neighbor. It is done using two rounds of beacon packets. Employing this protocol, a node is not allowed to communicate with the 2-hop neighbor directly. However, it reaches in 2-hops via the 1-hop neighbors. To determine the 2-hop forwarding set, the VAPOR first introduces three definitions such as a set of forwarding neighbors of node $x,\left\{S_{N} x\right\}$, Set of node $x$ 2-hop forwarding neighbors, $\left\{S_{2 N} x\right\}$, and Set of 2-hop selected forwarding candidates $\left(S_{(F 1, F 2)} x\right)$. The scenario for forwarding set is shown in Figure 1. In that, the dark shaded region represents the 1-hop neighbor forwarding area that is based on the destination direction. These three sets of nodes are estimated using Equations ((1)-(3)) when a sender node demands communication with a particular destination.

The $S_{N} x$ is defined to represent the set of neighboring nodes within the angle $(\alpha)$, and the distance between the nodes is denoted by $d(x, y)$ and assume that the area of circle $\chi$ is limited to a particular angle $(\angle \alpha)$ to form the 2-hop forwarding set that reduces the packet forwarding region [22].

$$
S_{N} x=\{y \mid(d(x-D)>d(y-D)), y \in \angle \alpha \chi(x)\} .
$$

The $\left(S_{2 N} x\right)$ is defined as a set of 2-hop neighbor nodes of node $x$ and is shown in the Equation (5).

$$
S_{2 N} x=\left\{z \mid y \in S_{N} x,(d(y-D)>d(z-D)), z \in \angle \alpha \chi(x)\right\} .
$$

The $S_{(F 1, F 2)} X$ is defined to represent the set of 2-hop forwarding members of node $x$.

$$
S_{(F 1, F 2)} x=\left\{(x, y) \mid y \in S_{N} x, z \in y \| z \in S_{2 N} x\right\} .
$$

From the definitions, it is clear that each node in 1-hop and 2-hop forwarding neighbor set relies on the communication range, but, the potential forwarding members set relies on the forwarding probability. In case, a node with an empty forwarding list within a communication range limited to a certain angle, the sender node adjusts the angle up to $90^{\circ}$ for increasing the probing area of a hello packet. However, the consideration of fixed 2-hop information for packet relaying leads to an unknown communication hole in a highly sparse network topology. VAPOR needs the support of the lower layers to avoid the communication hole. The link stability and capacity estimation scheme executes accurate 2-hop information for all the 2-hop forwarding set of the lower layers. However, in a highly dense network, the hop forwarding set leads to unnecessary routing overhead on each node. VAPOR applies the forwarding score measurement on 2-hop forwarding set when the network is highly sparse, otherwise, it takes into account 1-hop neighbor set of opportunistic routing.

\subsection{Selection of Best Forwarder and Forwarding Candidates}

The main problem associated with the QoS opportunistic routing is the selection and prioritization of high-quality forwarding nodes, due to the network dynamism. The greedy based opportunistic routing is not an optimal solution for selecting the high-quality forwarding nodes. The VAPOR selects the forwarding candidates prioritized

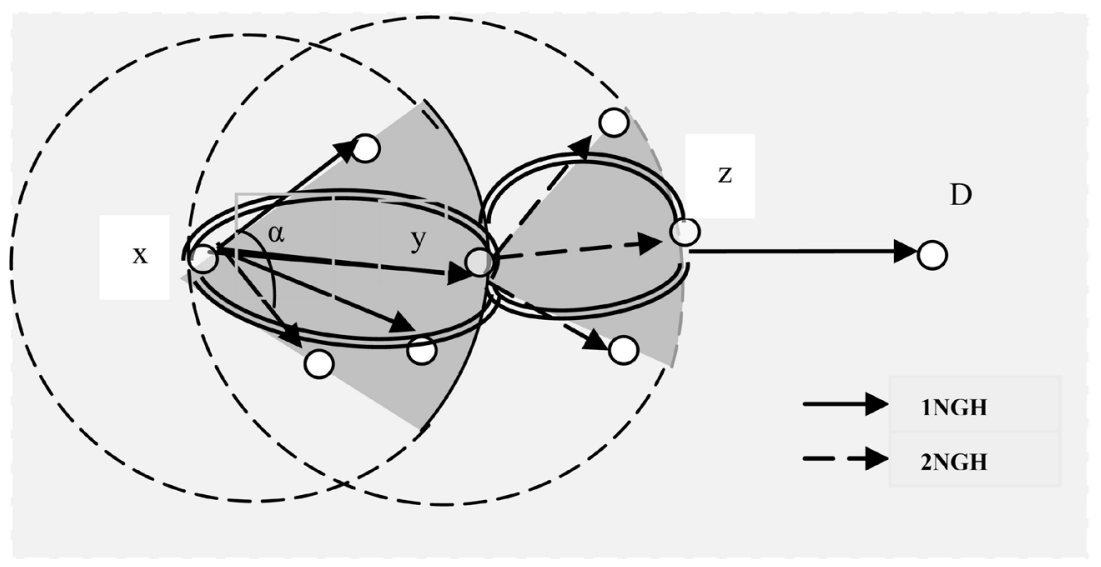

Figure 1. Forwarding set. 
by the factor such as the link quality, the capacity, and the connectivity factor. The high-quality forwarding candidates are selected within the area enclosed by a particular angle to increasing the reliability [22].

\subsubsection{Forwarding Probability for Candidates}

The nodes' forwarding probability is estimated according to the node's forwarding score using link stability and capacity, and connectivity factor. The RPSS in the physical layer supports the measurement of the link stability, and MAC layer supports the measurement of capacity. For the dense network, each node estimates the link stability time and capacity with its one-hop neighboring nodes. However, in sparse network VAPOR extends the measurements to 2-hop connections by using two rounds of beacon packets and applies the minimum value of stability and capacity in forwarding probability measurement. This work considers latter one in the estimation of node forwarding probability.

The sender node determines the route to reach the destination by scanning the $S_{2 N} X$, and alleviate the void due to the advanced prediction of 2-hop neighbor nodes. The sender node selects the highly resourceful nodes from the $S_{(F 1, F 2)} X$ set of forwarders according to the node stability, capacity, and void probability. The forwarding nodes from the $S_{(F 1, F 2)} X$ with the highest forwarding probability is measured in terms of node forwarding score, and connectivity factor that is inversely proportional to the void probability in the neighboring area are selected as forwarding candidates.

$$
\begin{gathered}
\text { Forwarding probability }=(\text { Node Forwarding Score } \times \text { Connectivity Factor })_{x}^{(y-z)} \\
\text { Node Forwarding Score }=(\text { Transmission Capacity })_{\text {Link Stability }}
\end{gathered}
$$

\section{1) Link Stability Estimation}

Considering the bidirectional communication, two nodes are placed within the range of each other. Each node sends the hello packet to its 1-hop neighbor nodes and also each receiver sends hello packets periodically enclosing its information (for every $\Delta t$ time). Node $x$ samples the RPSS of a hello packet received from the node $y$. It monitors the variation in the RPSS ( $\triangle R P S S$ ) of a received packet for every $\Delta t$ time. The received RPSS is higher when the distance between the mobile nodes are closer and vice versa [21].

\begin{tabular}{ll}
\hline$\triangle R P S S_{(x, y)}^{(1, t 2)}>0$ & Node $x$ and $y$ are closer at time $t 2$ than $t 1$ \\
$\Delta R P S S_{(x, y)}^{(1, t 2)}<0$ & Node $x$ and $y$ position does not change/nodes move in same direction with similar speed \\
$\Delta R P S S_{(x, y)}^{(t, 12)}=0$ & Node $x$ and $y$ are moving away from each other at $t 2$ \\
\hline
\end{tabular}

Let $\operatorname{RPSS}_{(x, y)}^{t 1}$ is the received hello packet signal noticed by a node $x$ with respect to the node $y$ at time $t 1$ and $t 2$.

$$
\left[\operatorname{RPSS}_{(x, y)}^{t 1}-\operatorname{RPSS}_{(x, y)}^{t 2}\right] / \Delta t \quad \text { where } y \in S_{N} x \text {. }
$$

Then, node $y$ estimates the link stability time of its 1-hop neighbor $z$ is shown in the Equation (9).

$$
\Delta \operatorname{RPSS}_{(y, z)}^{(t, t 2)}=\left[\operatorname{RPSS}_{(y, z)}^{t 1}-\operatorname{RPSS}_{(y, z)}^{t 2}\right] / \Delta t \quad \text { where } z \in\left[\left(S_{2 N} x\right) \& S_{N} y\right]
$$

To estimate the Link Stability time $\left(L S_{t}\right), D(x, y)^{t}$ denotes the distance between node $x$ and $y$ at time $t$, and $V(x, y)^{t}$ denotes the node speed on the network. The time taken by the nodes $x$ and $y$ to meet at their closest points $\left(L S_{\text {meet }_{(x, y)}}\right)$ is given as,

$$
\left(L S_{\text {meet }(x, y)}\right) t=D(x, y)^{t} / V(x, y)^{t} .
$$

After a node $x$ meet node $y$, they depart away from each other. The link is broken, when they cross over the communication range of each other is given as,

$$
\left(L S_{\text {cross }(x, y)}\right) t=\mathcal{R} / V(x, y)^{t} .
$$

Therefore, the total node stability time for positive $\triangle$ RPSS is,

$$
(+\Delta R P S S), L S_{t}=\left[D(x, y)^{t}+\Re\right] / V(x, y)^{t} .
$$


Moreover, for negative value of $\triangle \mathrm{RPSS}$,

$$
(-\triangle R P S S), L S_{t}=\left[\Re-D(x, y)^{t}\right] / V(x, y)^{t} .
$$

Figure 2 shows the mobile nodes $x, y$, and $z$ 's mobility direction. The node $x$ and $y$ move away from each other, while the nodes $y$ and $z$ meet at one point and later on move away from each other. Thus, the 2-hop link stability is,

$$
\begin{gathered}
L S_{(x, y)} t=\left(\Re-D(x, y)^{t}\right) / V(x, y)^{t} \\
L S_{(y, z)} t=\left\{\left(D(y, z)^{t}\right) / V(x, y)^{t}+\mathfrak{R} / V(x, y)^{t}\right\} \\
L S_{2 N} x=\min \left(L S_{(x, y)} t, L S_{(y, z)} t\right) .
\end{gathered}
$$

From the Equation (17), the 2-hop stability of each 2-hop connection is defined as the minimum link duration of a connection from forwarding set. The $L S_{2 N} X$ represents the 2-hop link stability, which is the maximum possible length of data transfer. The link stability measure increases the accuracy of 2-hop forwarding set in VAPOR.

\section{2) Link Capacity Estimation}

Consider each 2-hop forwarding set that includes $L 1$ and $L 2$ links. The capacity of the links in the 2-hop forwarding set is represented as $B_{x}\left(B_{1}\right.$ and $\left.B_{2}\right)$. The capacity of the forwarding set $\left(B_{f} x\right)$ is the minimum value of the links that constitute the 2-hop forwarding set. The estimation of nodes link capacity relies on the information available at the MAC layer. The MAC busy (M_busy), MAC idle (M_idle) period, Queue_packet waiting $(\tau)$ period, and the number of successive transmissions $\left(T_{s}\right)$ within an interval $\mathrm{d}$, are the MAC layer properties used to measure the link capacity $\left(B_{x}\right)$. In the equation (18), $\alpha$ is a constant value.

$$
\begin{gathered}
B_{x}=\left\{\alpha *\left[\left(T_{s} * M_{\text {idle }}\right) /\left(M_{\text {busy }} * d\right)\right]-(1-\alpha) *(q / d)\right\} \text { where } x=1 \text { and } 2 \\
B_{f} x=\min \left(B_{1}, B_{2}\right) .
\end{gathered}
$$

\section{3) Forwarding Score}

The link forwarding score measured for all 2-hop forwarding set ( $f$ ) of a sender node $x$ is shown in the Equation (20). It does not take additional time for recovering the new link for data forwarding since each node maintains the 2-hop forwarding list. The above concept is used for determining the capacity of each link. In that, $B_{\text {avg }}$ is the average bandwidth availability in each link.

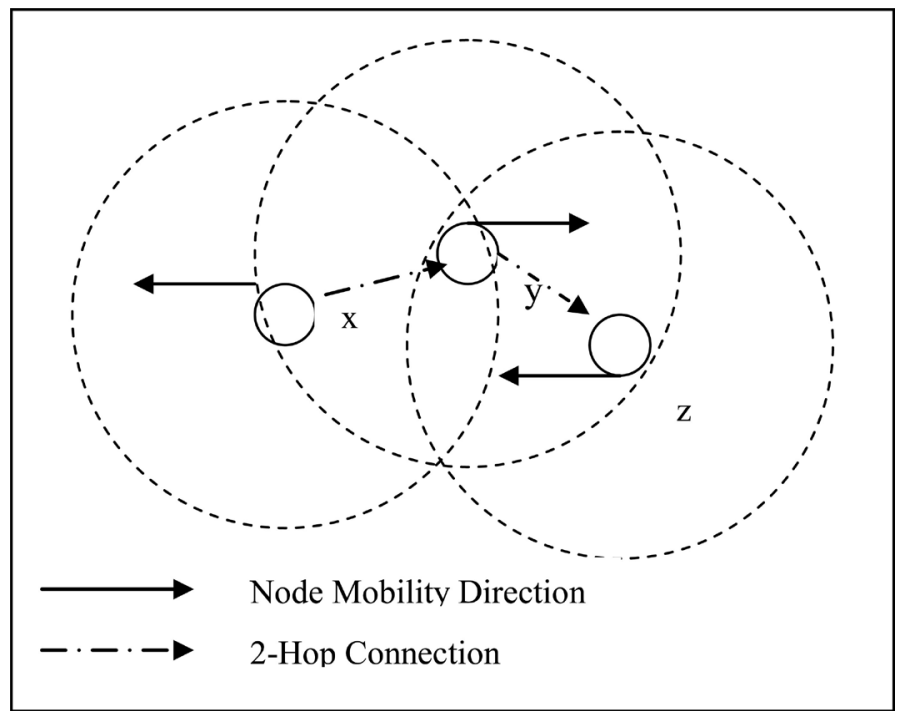

Figure 2. Node mobility direction. 


$$
\text { (Forwarding Score })_{x}^{y}=\left[\left(L S_{t}\right)\right]_{f} * B_{f} x+\left[x \cdot \max \left(L S_{t}\right)_{f}-\left(L S_{t}\right)\right] * B_{a v g} .
$$

Hence, the forwarding score is high for $(x, y)$. The VAPOR routing takes node connectivity factor in alleviating the communication hole, besides, considering the node capacity and stability of each node $\in S_{(F 1, F 2)} X$.

\section{4) Connectivity Factor $\left(C_{f}\right)$}

The connectivity factor $\left(C_{f}\right)$ represents the relationship between the node connectivity and the number of neighboring nodes in the forwarding set [23]. The connectivity factor is a main metric for selecting the forwarding nodes, and it is inversely proportional to the node density. If the node density is low, the connectivity factor decreases the forwarding probability or it increases the void probability. The probability of data forwarding increases, when the node density increases. The connectivity metric used to estimate the connectivity factor is $5.1774 \log N$, where $N$ is the number of nodes in the network. If each node is connected with more than 5.1774 $\log N$ number of neighboring nodes, then the probability of void creation is very less as the node connectivity increases.

$$
C_{f}=(5.1774 \log N /|N(n x)|) .
$$

From the Equation (21), if $|N x|$ is less than $5.1774 \log N$, the node $x$ is in the sparse area network. Thus, the probability of void creation is high and, as a result, it reduces the node connectivity factor. Moreover, if 5.1774 $\log N$ is less than the $|N x|$, the $C_{f}$ value is very low. Thus, the node connectivity is high, and the void probability is low when the node mobility rate is low.

\subsubsection{Prioritization of Forwarding Candidates}

A 2-hop forwarding set is selected as the forwarding nodes with the probability of $F_{p}$ is given as,

$$
F_{p}=10^{m} *\left(\left(F_{s}\right)_{x}^{y-z} * C_{f}^{x}\right) / \sum_{j=1}^{F}\left[\left(F_{s j}\right) * C_{f}^{j}\right] .
$$

The forwarding probability of a node is multiplied by $10^{m}$ where, $m$ varies from 1 to $n$. It is used specifically to transform the values into the integer values between 0 and 10 for reducing the storage overhead at each node [24]. The proposed work selects a high probability node for data forwarding. The sender node sets the timer for the forwarding node based on the value of $\min \left(L S_{t}\right)$ to improve the efficiency of the void prediction scheme and to transmit the data packets at a rate of $B_{f} x$. Once, the timer expires the sender node selects the high priority node from the recently updated 2-hop forwarding set. A node with a high probability within the forwarding region gets higher priority for data forwarding and is named as the best forwarder and the others as forwarding candidates.

\subsection{Data Forwarding}

VAPOR protocol follows an opportunistic routing, instead of using the distance metric. VAPOR exploits the forwarding probability in terms of multiple quality metrics to select candidates and prioritize them. The candidates in the forward list of data packets in the order of its probability. The best forwarder has the highest priority to forward the data packet. The next priority forwarding candidate gets the turn to data forwarding when the best forwarder fails to transmit the packet. If the forwarding candidate fails to hear the data packets transmitted by the best forwarder, it induces the duplicate transmission. The VAPOR sends the data packets in a batch operated mode to limit the duplicate transmission. The link stability period is the maximum possible duration of data transmission by a next hop. The source node removes 2-hop forwarding set from the list and selects a new set for transmitting the subsequent packets at the end of link stability period of the next-hop node.

Figure 3 illustrates the basic operation of VAPOR. The best forwarder A transmits the data packet in a normal state, and it suppresses the forwarding candidates B and C. However, node B and C overhears the best forwarder's transmission. If the best forwarder fails to transmit the data packet for a particular time slot, the highest priority forwarding candidate B selects a node from the interference range of node A. In Figure 3(b), the forwarding candidate B selects node $\mathrm{E}$ to transmit the packet. The identification of duplicate packet transmission is not feasible if the forwarding candidates forward the data packet in a highly sparse network topology. The node B selects node $G$ from the forwarding candidate list of best forwarder A, and transmits the packet as unicast. After receiving the packet from node B, G ensures from the packet list, whether it has received the same packet from the other nodes. 


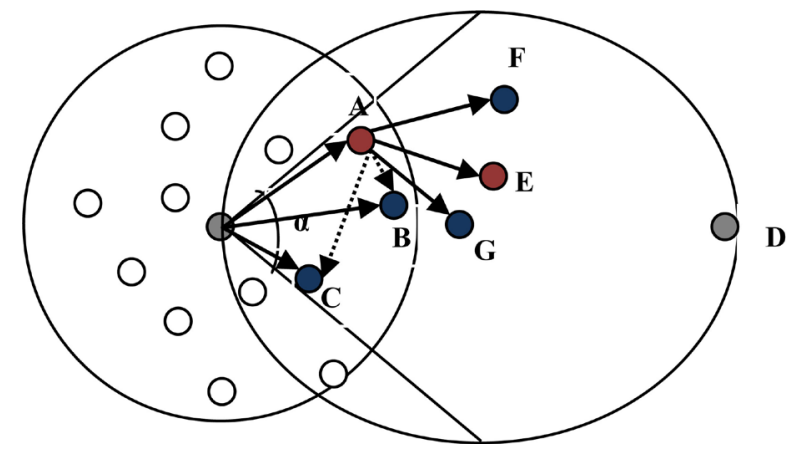

(a)

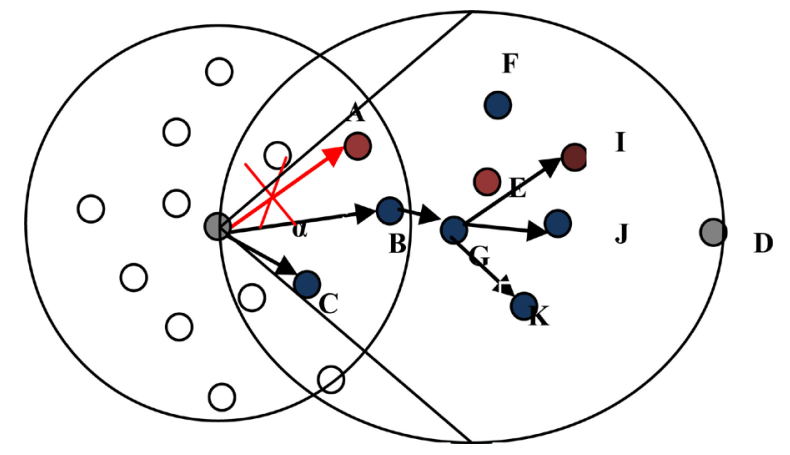

(b)

Figure 3. VAPOR operation. (a) Best forwarder transmission; (b) Forwarding candidate transmission.

\section{Batch Operated Transmission}

A set of data packets is grouped into batches, and all the data packets in the same batch are included in the forwarder list when it is transmitted from the source node [3]. The set of nodes that receive the packet retain the information about the forwarding list, batch ID, packet forwarding area, and transmission time. In addition, it maintains the list of packets that has the same batch ID. Each packet includes the packet map for corresponding batch ID, which marks the sequence number of the packets that has the same batch ID and that have been already transmitted by the node with higher probability. It waits for the reception of all the packets with the same batch ID and the next priority node forwards the data packets that are not listed in the packet map of the recently overheard packet. At the end of the batch, the missing packet in a batch and the next batch of packets being transmitted concurrently avoids delay induced by the batch forwarding. The missing packets are transmitted as unicast by the next priority candidate while the next batch of packets is transmitted by a recently selected best forwarder in an opportunistic scenario. Thus, the highest priority forwarding candidate transmits the remaining packets, thereby improving the end-to-end data delivery. The VAPOR utilizes the advantage of batch operated data transmission, only when the recently updated packet is overheard.

\subsection{Forwarding Candidate Coordination for Duplicate Control}

The propagation area of the data packet is limited to a certain angle $(\alpha)$ between the source and the destination. The centralized coordination among the forwarding candidates needs to schedule the data transmission to reduce the duplicate relaying. The best forwarder that has received packets from the sender node forwards them first towards the destination. It indicates the missing packets to the lower priority forwarding candidates, by attaching batch map in each packet. The higher priority forwarding candidate waits for a particular time slot to hear the transmitted packet. If it fails to hear the transmission of the best forwarder, the packets sent by the forwarding candidate are identified as duplicate. In the VAPOR, the forwarding candidates do not have the same forwarding scenario as the best forwarder, eventually, it is forced to select a node from the forwarding list of the best forwarder. Thus, the selected node within the forwarding list of the best forwarder identifies and drops the duplicate packets successfully. Thus, the VAPOR ensures an efficiency of multi-objective QoS routing in terms of network throughput and reliability.

\section{Analysis of the VAPOR}

\subsection{Throughput versus Scalability}

In a large-scale network, the use of highly stable links for data forwarding minimizes the packet drop, thereby increasing the network throughput. When the data forwarding nodes change dynamically due to the unpredictable node mobility, the frequent link disconnections lead to significant packet drops during the data transmission.

The throughput depends on the number of nodes involved in the data transmission and the packet loss induced by the intermediate node's mobility. The estimation of the average number of hops $(h)$ between the source and the destination is as follows:

$$
h=D / l .
$$


To estimate the average h between the source $\mathrm{S}$ and the destination $D$, determine the average number of a 1-hop neighbor of a node $(\eta)$ and the average 1-hop length $(l)$. The average 1-hop length can be estimated as the average distance between the $\mathrm{S}$ and its neighbor nodes within the communication range $(\Re)$.

$$
\begin{gathered}
\eta=\left(N * \Re^{2} / H * W\right) \\
l=1 / \eta \sum_{j=1}^{\eta} d(s-j) .
\end{gathered}
$$

Substituting (24) and (25) in (23), the expected number of hops between source and destination is estimated as follows.

$$
h=D /\left((H * W) / \pi \Re^{2}\right)\left[\sum_{j=1}^{\eta} d(S-j)\right] .
$$

Therefore, the achieved throughput $\left(T_{o}\right)$ of the proposed system is given as,

$$
T_{o}=\left(\text { Generated packets }-n\left(P_{d} h\right) * \text { packet size }\right) / \text { second } .
$$

In the Equation (27), $n\left(P_{d} h\right)$ represents the packet drop at all the $h$ for the change of every node $n$. The reduction of the packet drop is due to the selection of highly stable nodes for data forwarding. Thus, the proposed system increases the network throughput significantly even on a large scale network.

\subsection{Delays versus Node Density}

The node density $(\rho)$ can influence the performance of an opportunistic routing system in terms of End-to-End Delay $\left(D_{o}\right)$. When the number of nodes in the network is low, the delay of packets tends to be high. The maintenance of optimal routes in advance reduces the delay of VAPOR, but it tends to be an unnecessary delay in the dense network. To reduce this, VAPOR changes the routing mode in which the nodes do not involve the 2-hop neighbor set in selecting the forwarding candidate.

$$
D_{o}=\frac{d(S, D)}{c}+\sum_{h_{n}=1 m=1}^{\left|h_{n}\right| \Delta h_{n}}\left(\tau_{h_{n m}}^{\prime}\right) .
$$

The routing delay depends on the path length, the packet speed $(c)$, the number of times a node changes its next hop $\left(\Delta h_{n}\right)$ during packet forwarding, and the time taken to change the next hop $\left(\tau^{\prime}\right)$. The estimation of the average number of hops $(h)$ between the source and the destination is as shown in the Equation (26). The $\tau$ is inversely proportional to the node density, because in sparse network the computational time for forwarding candidates is high. The sparse network has less $\Delta h_{n}$, whereas in a dense network it is high. Thus, VAPOR optimally balances the routing delay and the packet reliability in both the sparse and the dense network.

\subsection{Duplicate Relaying vs Mobility and Traffic}

A greedy selection is not an optimal solution always for selecting the forwarding node, as it moves quickly out of the communication range. Owing to the node mobility, it is not feasible to deliver the data packets promptly. In most cases, the forwarding candidates fail to overhear a packet transmitted by a next hop node during mobility. The main concern of opportunistic routing is the duplicate relaying. There are two possible cases in which the duplicate packets occur.

1) A packet sent from a moving node is successfully received, but it might not be overheard by the forwarding candidates.

2) Due to high traffic, forwarding candidates fail to overhear a packet transmission.

$$
P_{d p}=P_{m} * P_{t} \text {. }
$$

In the Equation (29), $P_{d p}$ represents the probability of duplicate packet transmission and the $P_{m}$ is the probability of the packet delivered successfully from $\mathrm{S}$ to $\mathrm{A}$, but not overheard by the forwarding set. The $P_{t}$ represents the probability of duplicate packets due to network dynamics. To reduce the duplication, VAPOR selects highly stable nodes instead of a greedy node and employs the batch operated transmission. Consider the total number of packets is divided into $B$ batches and each batch contains $C$ packets. 


$$
\text { Total packets }=B * C \text {. }
$$

Assume that the forwarding candidate is not able to overhear due to node mobility $(\gamma)$ with the probability of $P_{o}$.

$$
P_{m}=1-\left(1-P_{o}\right)^{\gamma} .
$$

Due to the selection of highly stable routes and the measurement of link lifetime, the $P_{m}$ is negligible in VAPOR. The network traffic may increase the $P_{t}$, but the batch operated transmission reduces the $P_{t}$ more than the existing work. The $P_{c}$ denotes the probability that the inaccuracy of the batch map in the recently overheard packet with the same batch $\mathrm{B}$ as shown in the Equation (32).

$$
\begin{aligned}
& P_{t}=1-\left(1-P_{o}\right)^{t} * P_{c} \\
& P_{c}=1-\left(O C_{b} / T R_{b}\right) .
\end{aligned}
$$

The inaccuracy of recently overheard packet's $\left(O C_{b}\right)$ batch map is fixed with the number of transmitted packets $\left(T R_{b}\right)$ over the total packets.

$$
P_{d p}=\left\{1-\left(1-P_{o}\right)^{t} *\left\{1-\left(O C_{b} / T R_{b}\right)\right\}\right\} .
$$

Substituting (31), (32), and (33) in (29), the probability of duplicate packet transmission is estimated. Thus, the batch operated transmission of VAPOR significantly decreases the duplication even in a high traffic and dynamic network topology.

\subsection{Storage Overhead vs Node Density}

In a low-density network, the proposed protocol VAPOR broadcasts the beacons to identify the 1-hop and the 2-hop neighbor nodes. To retain the 2-hop information, the VAPOR employs two rounds of beacon messages. In the first round, every node informs its location to the 1-hop neighbor nodes. Then, it triggers another round of beacon to obtain the 1-hop neighbor information. Therefore, routing overhead occurs twice in the network as shown in the Equation (35).

$$
C_{\text {overhead }}=(2 N \eta)^{\rho}
$$

Moreover, the maintenance of 2-hop neighbor set increases the storage overhead. In a MANET, maintaining the neighbor list in a table is essential. Therefore, this issue needs to be considered due to the limited memory and energy of the mobile nodes.

$$
H=\left[\begin{array}{cccc}
N_{(A, B)} & N_{(A, B+1)} & \cdots & N_{(A, B+n)} \\
N_{(A+1, C)} & N_{(A+1, C+1)} & \cdots & N_{(A+1, C+n)} \\
\vdots & \vdots & & \vdots \\
N_{(A+n, E)} & N_{(A+n, E+1)} & \cdots & N_{(A+n, E+n)}
\end{array}\right]
$$

Each node maintains the 2-hop neighbor set in a matrix $\mathrm{H}$ to reduce the recovery time and update the 2-hop forwarding set. In $\mathrm{H}$ matrix, A represents the 1-hop neighbors and B represents 2-hop neighbors set of node N. To minimize the storage overhead, the forwarding probability value is converted into the integer value between 0 and 10 . The unsigned integer value between 0 and 10 takes only 4 bits of memory space. Thus, it saves $50 \%$ of memory. Moreover, in a high-density network the routing overhead induced by the VAPOR that employs the 1-hop neighbor information for routing decision is reduced to $C_{\text {overhead }} / 2$.

\section{Performance Evaluation}

NS-2 based simulation shows the performance of the proposed VAPOR protocol. The proposed VAPOR is compared with the existing POR. This simulation study concentrates the QoS parameters such as throughput, delay, reliability, and the overhead of VAPOR. 


\subsection{Simulation Model}

For the simulation of varying mobility, traffic, density, and scalability, consider the randomly positioned mobile nodes of 50,100,150, 200, 250 and 300 within the $1000 \times 1000 \mathrm{~m}^{2}$ area. The moving velocity of a node varies between $5 \mathrm{~m} \cdot \mathrm{s}^{-1}$ to $45 \mathrm{~m} \cdot \mathrm{s}^{-1}$. It simulates an IEEE 802.11 MAC layer with a node communication range of 150 $\mathrm{m}$. The data packet size is 512 bytes. The network is simulated for 500 seconds.

\subsection{Simulation Results}

\subsubsection{Impact of Node Speed}

In this scenario, the network performance is evaluated by varying the node speed from 5 m/s to 45 m/s with 100 nodes. The throughput is defined as the number of bits delivered to the destination per second. From Figure 4(a), it is observed that the network throughput degrades while the speed of the mobile node increases. The proposed VAPOR includes the estimation of the link stability and the capacity that tends to determine the highly resourceful and the stable nodes to forward the data packets. The proposed VAPOR increases the throughput by

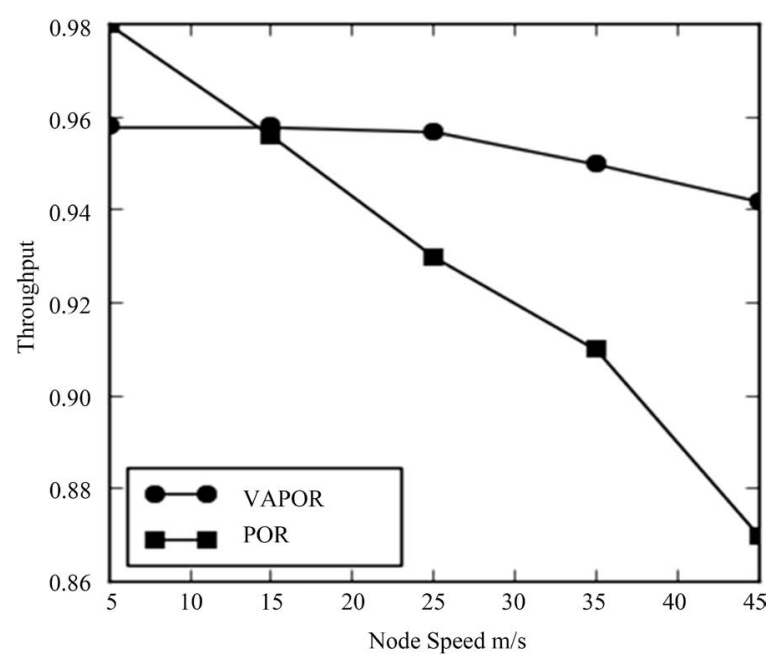

(a)

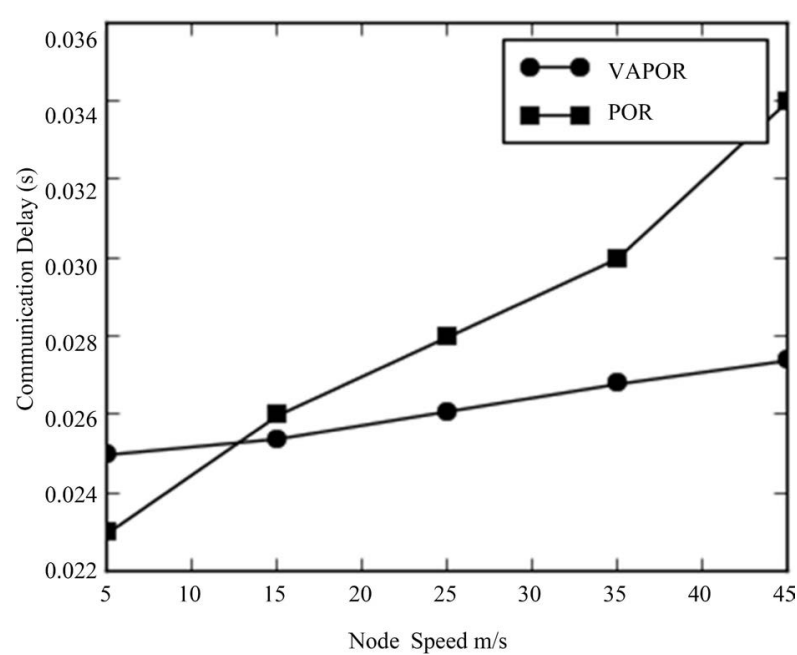

(b)

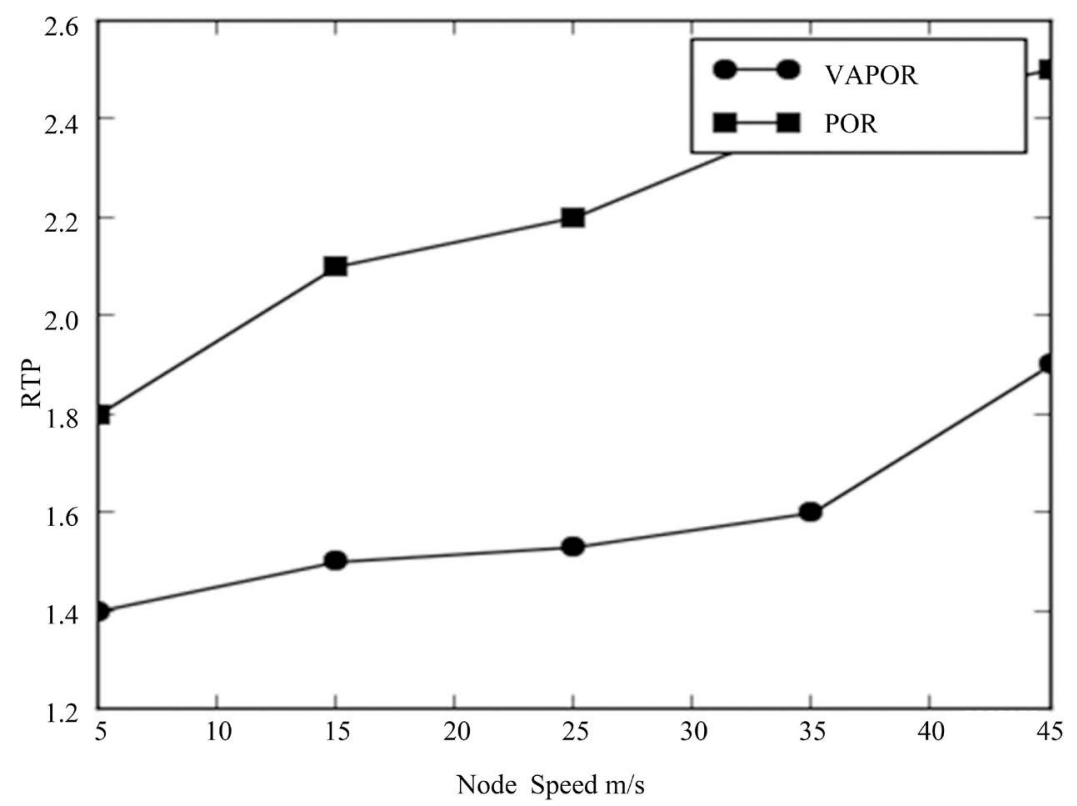

(c)

Figure 4. (a) Node speed vs. throughput; (b) Node speed vs. communication delay; (c) Node speed vs. RTP. 
$2 \%$ to $3 \%$ than POR at a speed of $25 \mathrm{~m} / \mathrm{s}$. Thus, the proposed VAPOR increases the packet forwarding efficiency than POR in high-speed scenarios.

From Figure 4(b), the delay of VAPOR increases as the node speed increases, whereas the delay of POR increases much faster. This result shows the effectiveness of the consideration of forwarding score in the design of forwarding set of VAPOR. Even when the node speed exceeds the $25 \mathrm{~m} / \mathrm{s}$, it nearly delivers most of the data packets at a time, but the communication delay of POR increase significantly. From the figure, it is observed that when the node speed is below $10 \mathrm{~m} / \mathrm{s}$, POR outperforms VAPOR, but the performance of POR degrades when the node speed keeps increasing. The main reason is the greedy node selection, which leads to frequent link disconnections when the node speed is too high. In Figure 4(b), VAPOR delivers a packet in the range of 0.026 Sec at the point of 35 data flows, but it is slightly higher than POR.

Figure 4(c) shows that the Receiving Time per Packet (RTP) which denotes the number of times the same packet (duplicate) received at the destination grows linearly with the node mobility. In VAPOR, the measurement of link lifetime reduces the value of the RTP, especially when the node mobility is high. When the forwarding candidate set is large, RTP increases gradually as expected in the face of highly dynamic network topology. In VAPOR, RTP increases in the range of $10 \%-11 \%$ when the node mobility increases in the range of 10,20 , 30 , and $40 \mathrm{~m} / \mathrm{s}$. The VAPOR reduces the RTP value by $7 \%-8 \%$ than the POR in a high mobility scenario.

\subsubsection{Impact of Traffic Flows}

The network traffic varies from 10 to 50 flows to evaluate the proposed protocol performance. Figure 5(a) demonstrates that VAPOR can scale to a high traffic network. The throughput of both the protocols remains close to 0.975 for 20 data flows. In POR, opportunistic routing introduces more channel contentions which significantly degrades the network throughput in high traffic scenario. For example, when the node traffic flow is 30 , its throughput is only 95\%. The POR performance indicates that the mechanism for coordinating the forwarding candidates is incapable of working efficiently in a high traffic environment. The VAPOR considers the link capacity in the design of the forwarding candidate set, and thus it avoids packet loss due to congestion.

From Figure 5(b), it is observed that in a high network traffic, POR fails to deliver effectively. When the number of data flows is 20 , the data packets delivered within a time are relatively poor compared to the VAPOR. The VAPOR reduces the communication delay compared to POR since the network traffic may increase the collision on the greedy node and the queuing delay. The impact of network traffic on the RTP is shown in Figure 5(c). The RTP increases in the range of $10 \%-16 \%$, when the network traffic is increased from 10 to 50 data flows. However the duplication still occurs in VAPOR, because it depends on the recently updated packet. In VAPOR, the RTP value is considerably reduced from 3\% - 6\% than the POR in a high traffic (30 data flows) condition.

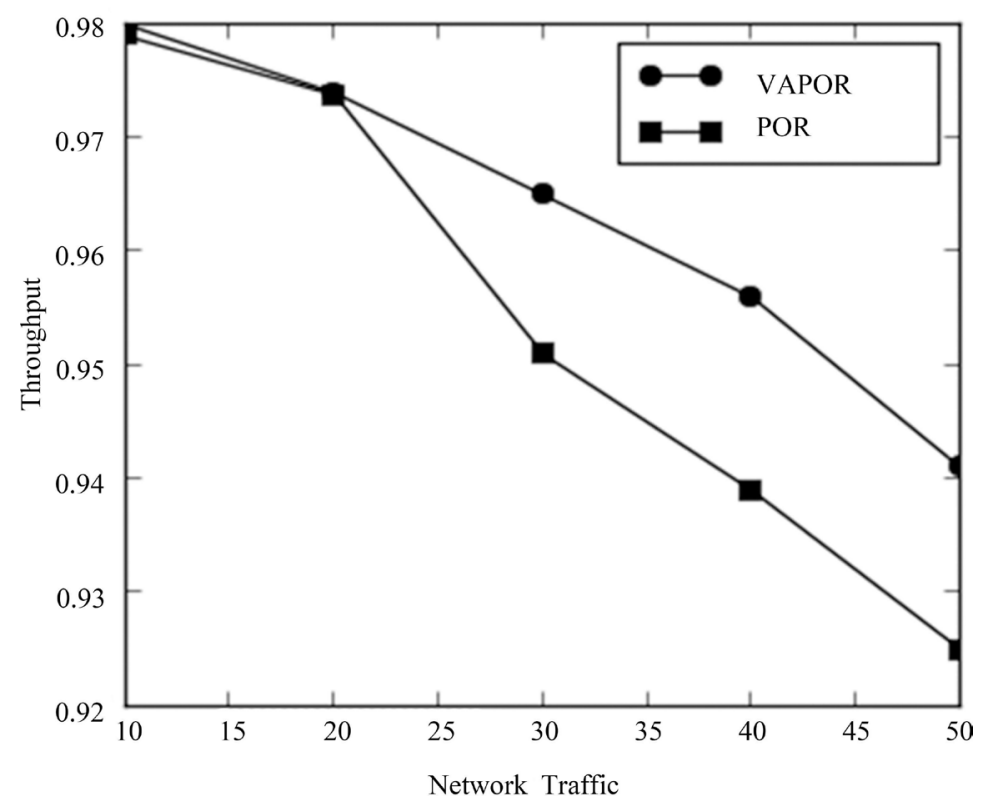

(a) 


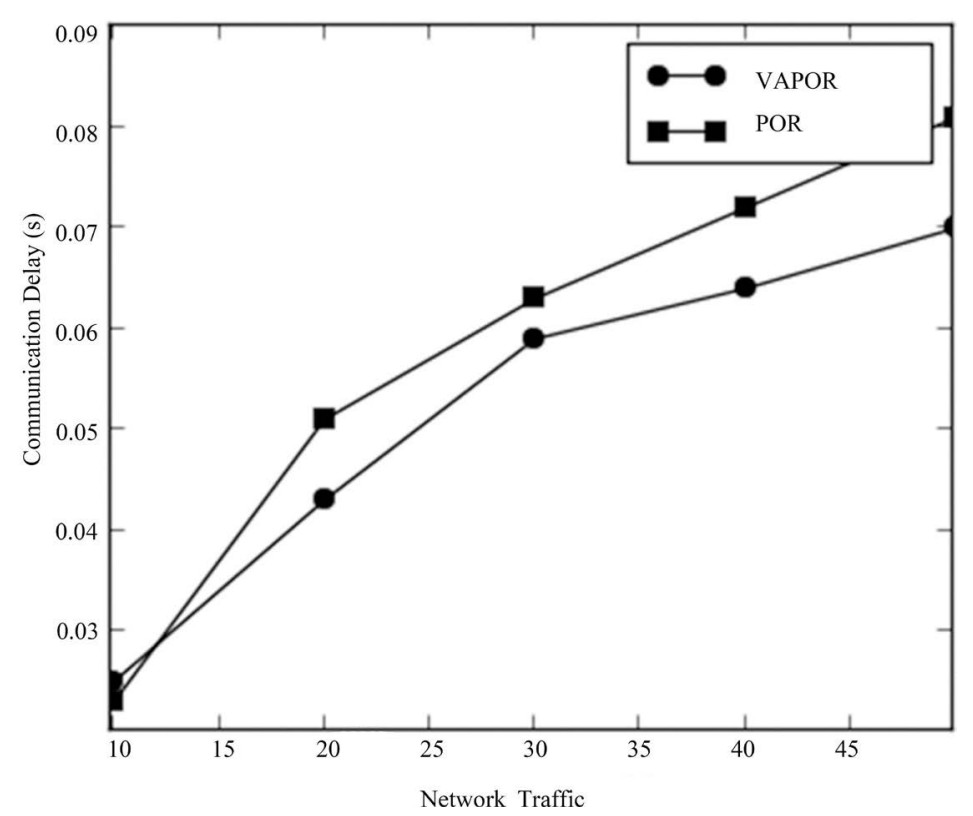

(b)

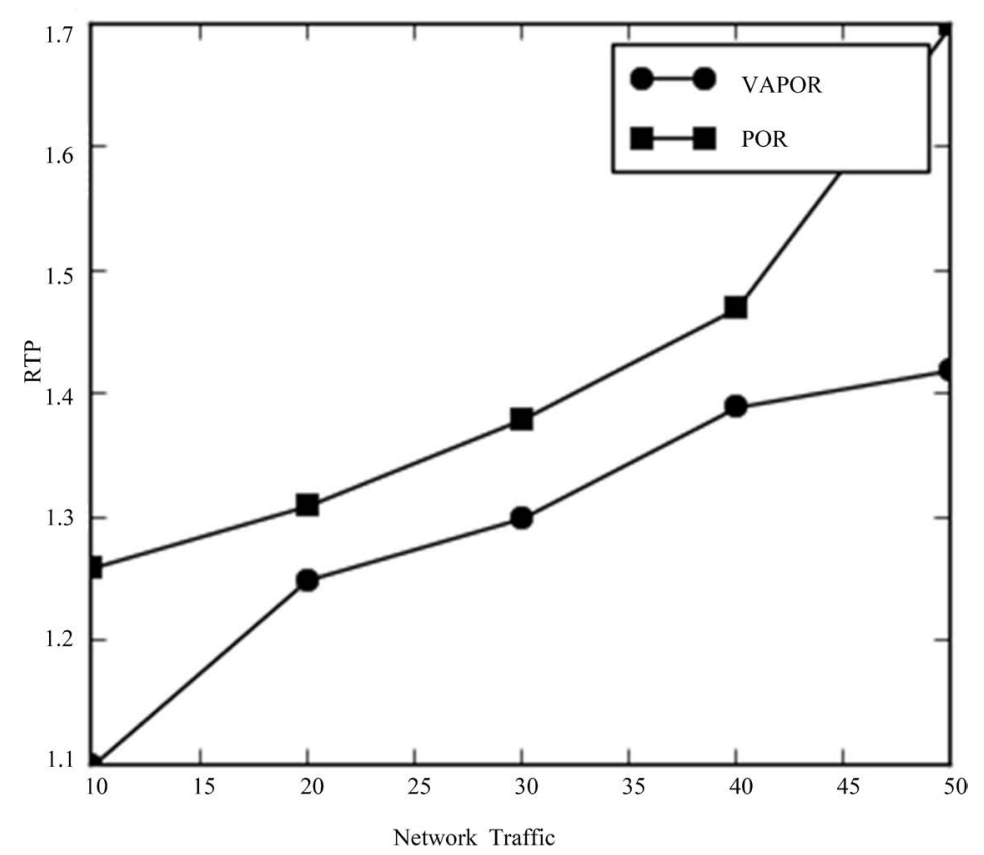

(c)

Figure 5. (a) Network traffic vs. throughput; (b) Network traffic vs. communication delay; (c) Network traffic vs. RTP.

\subsubsection{Impact of Node Density}

The Void Influence in Communication (VIC) denotes the delay of communication due to void. In the simulation, various node densities create different void probability. From Figure 6(a), it is observed that the VIC decreases with increasing node density. The main reason for the additional packet delay is that the sender node transmits the fixed rate of data packets towards the destination without the knowledge of the residual capacity. With the support of link stability estimation, the accuracy of void prediction and packet forwarding reliability is enhanced. The VIC is low in both VAPOR and POR when the node density is high since the possibility of a communication hole in the network is less. In the low-density condition, VAPOR performs superior due to the advance prediction of 2-hop information and each node is aware of the void, and hence the delay of VAPOR 


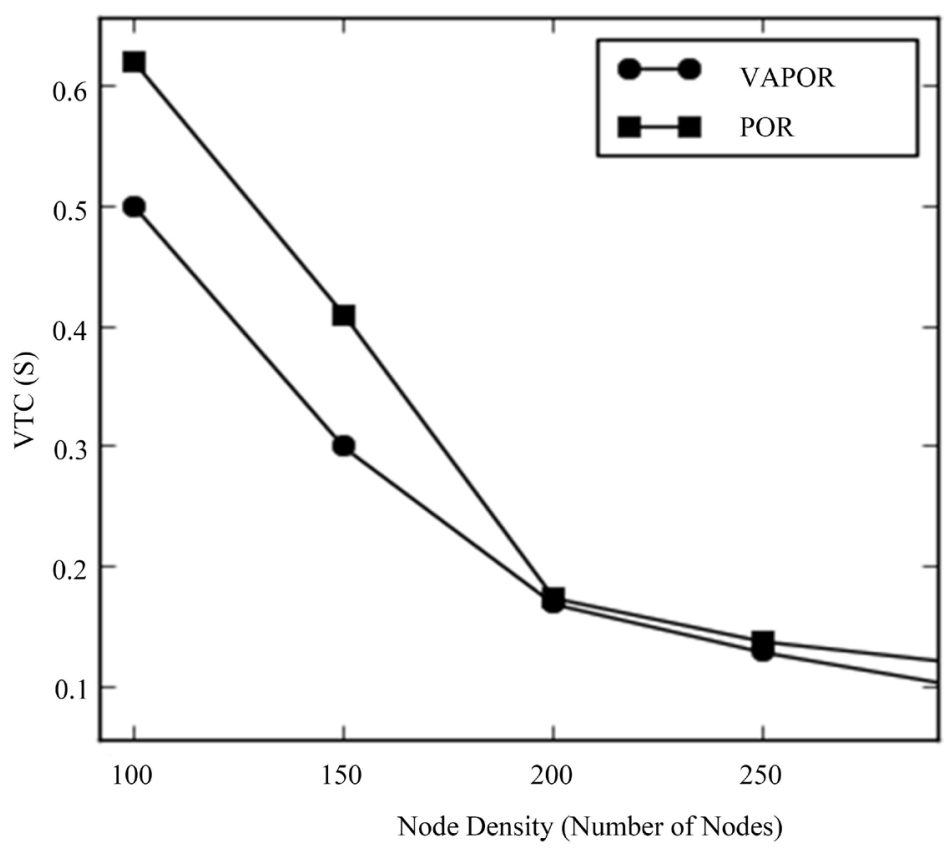

(a)

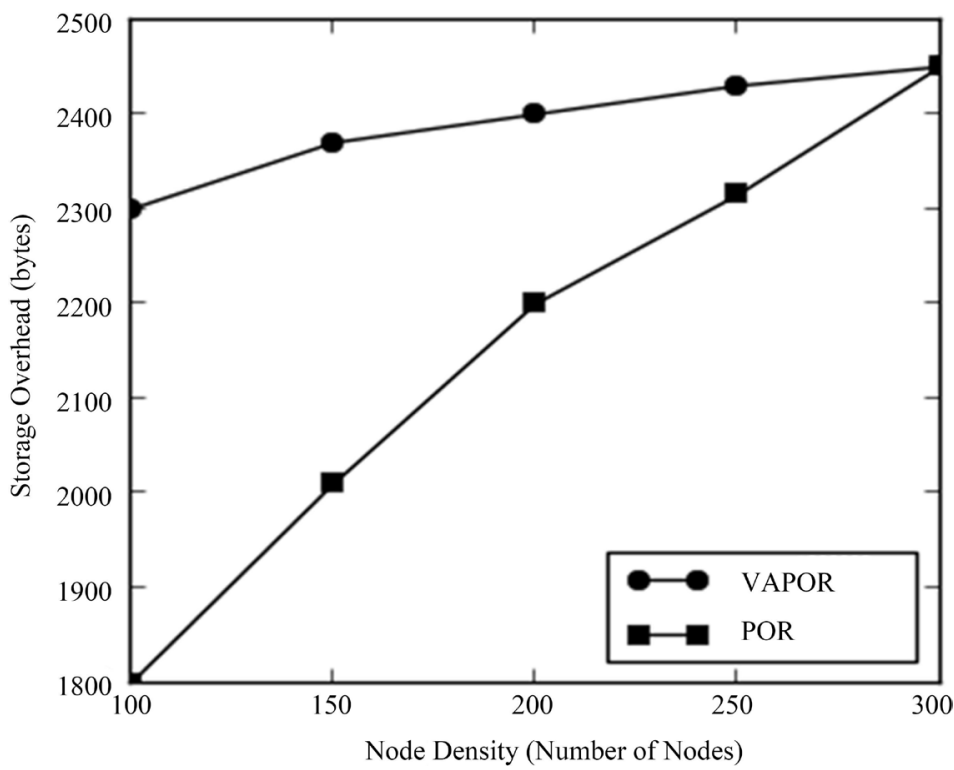

(b)

Figure 6. (a) Node density vs. VIC; (b) Node density vs. storage overhead.

gets reduced in the range of $20 \%$ than POR.

The geographic routing needs to store only the 1-hop neighbor list. However, the proposed work estimates and retains 2-hop neighbor nodes for alleviating the communication hole in a sparse network. Moreover, the storage overhead increases slowly, when increasing the node density as shown in Figure 6(b). It maintains only 1-hop forwarding set in a high-density network and converts the forwarding probability values $(0-1)$ into an unsigned integer value between 0 to 10. Moreover, the 1-hop/ 2-hop forwarding set and its forwarding score values are stored in a matrix. It leads to reduced storage overhead and retrieval time of routing information.

\subsubsection{Impact on Scalability}

The network size varies from $500 \mathrm{~m} \times 500 \mathrm{~m}$ to $2500 \mathrm{~m} \times 2500 \mathrm{~m}$ to evaluate the scalability of the proposed 


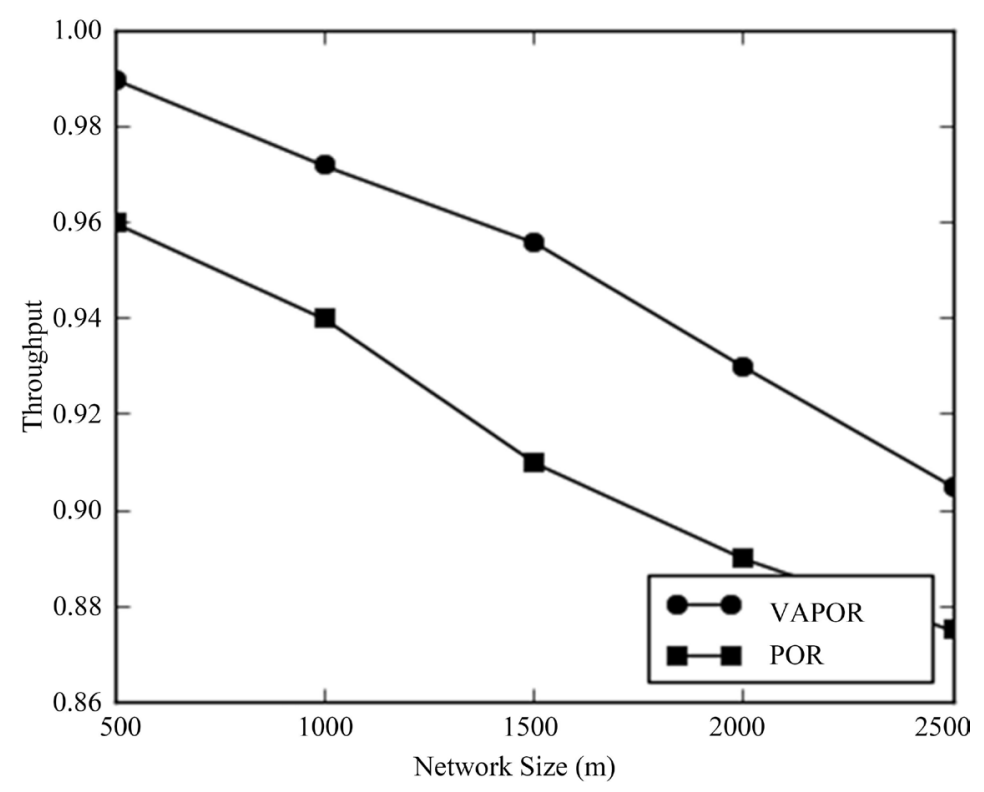

(a)

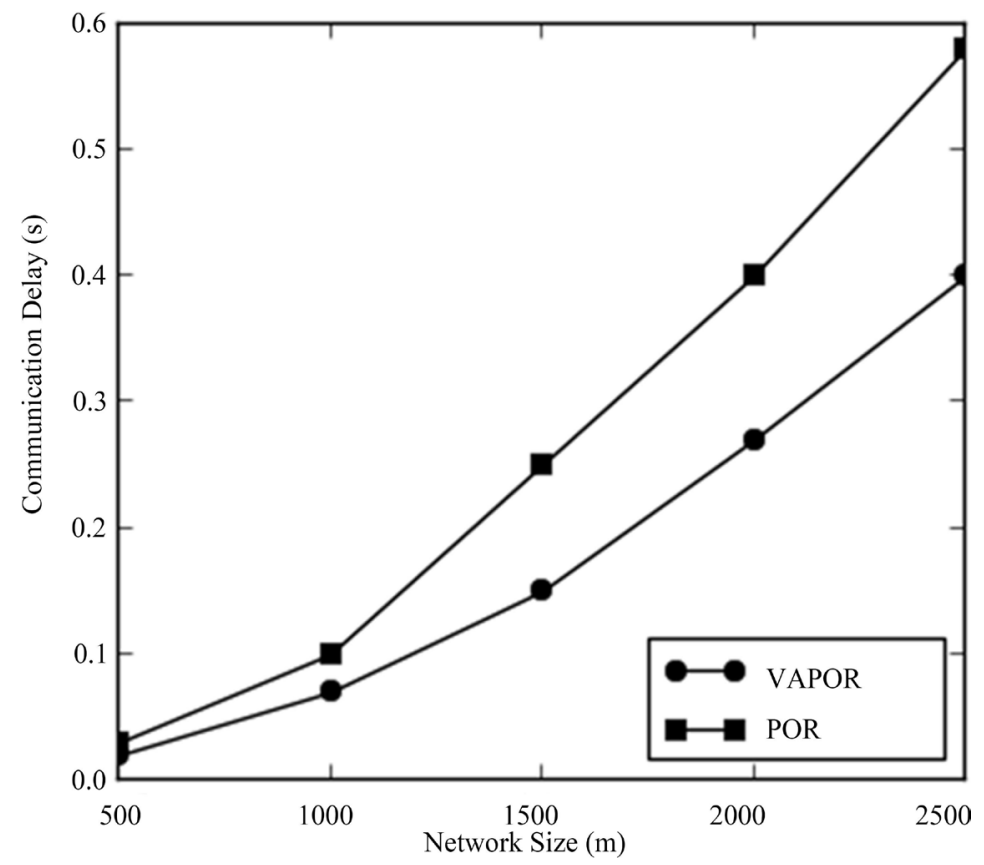

(b)

Figure 7. (a) Network size vs. throughput; (b) Network size vs. communication delay.

protocol. From Figure 7(a), it is observed that the VAPOR is scalable in a large scale network. The total number of nodes varies from 50 nodes to 1250 nodes to fix the node with the same density for all scenarios. When the network size is $2000 \mathrm{~m} \times 2000 \mathrm{~m}$ with 800 nodes, the difference between the throughput of POR and VAPOR is more than $9 \%$. As the network size increases, the path length and link disconnections also increase gradually. The VAPOR achieves scalability through a measure of the forwarding score, which takes the advantage of the link stability and the network capacity to simplify the mobility management and the congestion.

\section{Conclusion}

In this paper, an efficient VAPOR protocol is proposed to enhance the routing quality both in terms of the 
throughput and the reliability. The notion of the 2-hop information based routing technique delivers superior QoS both in the sparse and the dense network. The VAPOR effectively manages the communication hole and increases the network throughput. The method of the selection of candidates and void prediction schemes of the VAPOR ensures reliability and maximum packet delivery. The perfect coordination among the forwarding candidates minimizes the duplicate relay and as well as the wastage of the network resources. The advantage of the batch packet forwarding through the attached batch map in each data packet avoids further duplication. The integration of the storage overhead scheme ensures the timeliness of the data delivery and reduces retrieval time. The NS2 simulation results reveal that the proposed VAPOR is more suitable for QoS routing in terms of both throughput and reliability than the other geographic routing protocols in MANET.

\section{References}

[1] Stojmenovic, I. (2002) Position-Based Routing in Ad Hoc Networks. IEEE Communications Magazine, 40, $128-134$. http://dx.doi.org/10.1109/MCOM.2002.1018018

[2] Cadger, F., Curran, K., Santos, J. and Moffett, S. (2013) A Survey of Geographical Routing in Wireless Ad-Hoc Networks. IEEE Communications Surveys \& Tutorials, 15, 621-653. http://dx.doi.org/10.1109/SURV.2012.062612.00109

[3] Biswas, S. and Morris. R. (2005) EXOR: Opportunistic Multi-Hop Routing for Wireless Networks. Proceedings of the 2005 Conference on Applications, Technologies, Architectures, and Protocols for Computer Communications, 133-144. http://dx.doi.org/10.1145/1090191.1080108

[4] Abdrabou, A. and Zhuang, W.H. (2006) A Position-Based QoS Routing Scheme for UWB Mobile Ad Hoc Networks. IEEE Journal on Selected Areas in Communications, 24, 850-856. http://dx.doi.org/10.1109/JSAC.2005.863867

[5] Chen, D. and Varshney, P. (2007) A Survey of Void Handling Techniques for Geographic Routing in Wireless Networks. IEEE Communications Surveys and Tutorials, 9, 50-67. http://dx.doi.org/10.1109/COMST.2007.358971

[6] Hanzo, L. and Tafazolli, R. (2007) A Survey of QOS Routing Solutions for Mobile Adhoc Networks. IEEE Communications, 9, 50-70.

[7] Mueller, S., Tsang, R. and Ghosal, D. (2004) Multipath Routing in Mobile Ad Hoc Networks: Issues and Challenges. In: Calzarossa, M.C. and Gelenbe, E., Eds., Performance Tools and Applications to Networked Systems, Springer, Berlin Heidelberg, 209-234. http://dx.doi.org/10.1007/978-3-540-24663-3_10

[8] Zeng, K., Yang, Z. and Lou, W. (2009) Location-Aided Opportunistic Forwarding in Multirate and Multihop Wireless Networks. IEEE Transactions on Vehicular Technology, 58, 3032-3040.

[9] Leontiadis, I. and Mascolo, C. (2007) GeOpps: Geographical Opportunistic Routing for Vehicular Networks. 2007 IEEE International Symposium on a World of Wireless, Mobile and Multimedia Networks, Espoo, 18-21 June 2007, 1-6. http://dx.doi.org/10.1109/wowmom.2007.4351688

[10] Yang, S., Yeo, C.K. and Lee, B.S. (2012) Toward Reliable Data Delivery for Highly Dynamic Mobile Ad Hoc Networks. IEEE Transactions on Mobile Computing, 11, 111-124. http://dx.doi.org/10.1109/TMC.2011.55

[11] Rozner, E., Han, M.K., Qiu, L. and Zhang, Y. (2011) Model-Driven Optimization of Opportunistic Routing. Proceedings of the ACM SIGMETRICS Joint International Conference on Measurement and Modeling of Computer Systems, 229-240. http://dx.doi.org/10.1145/1993744.1993771

[12] Yang, S., Zhong, F., Yeo, C.K., Lee, B.S. and Boleng, J. (2009) Position Based Opportunistic Routing for Robust Data Delivery in MANETs. 2009 IEEE Conference on Global Telecommunications (GLOBECOM), Honolulu, Hawaii, USA, 30 November-4 December 2009, 1325-1330. http://dx.doi.org/10.1109/glocom.2009.5425351

[13] Wang, Z.H., Chen, Y.Z. and Li, C. (2012) CORMAN: A Novel Cooperative Opportunistic Routing Scheme in Mobile Ad Hoc Networks. IEEE Journal on Selected Areas in Communications, 30, 289-296. http://dx.doi.org/10.1109/JSAC.2012.120207

[14] Felemban, E., Lee, C.-G., Ekici, E., Boder, R. and Vural, S. (2005) Probabilistic QoS Guarantee in Reliability and Timeliness Domains in Wireless Sensor Networks. IEEE 24th Annual Joint Conference of the IEEE Computer and Communications Societies, 13-17 March 2005, 2646-2657. http://dx.doi.org/10.1109/infcom.2005.1498548

[15] Zeng, K., Lou, W., Yang, J. and Brown, D.R. (2007) On Throughput Efficiency of Geographic Opportunistic Routing in Multihop Wireless Networks. Mobile Networks \& Applications, 12, 347-357. http://dx.doi.org/10.1007/s11036-008-0051-7

[16] Shah, S.H. and Nahrstedt, K. (2002) Predictive Location-Based QoS Routing in Mobile Ad Hoc Networks. Proceedings of IEEE International Conference on Communications, 1022-1027.

[17] Karp, B. and Kung, H.T. (2000) GPSR: Greedy Perimeter Stateless Routing for Wireless Networks. Proceedings of the 6th Annual International Conference on Mobile Computing and Networking, 243-254. 
http://dx.doi.org/10.1145/345910.345953

[18] Raji, V. and Mohan Kumar, N. (2014) An Effective Stateless QoS Routing for Multimedia Applications in MANET, International Journal of Wireless and Mobile Computing, 7, No. 5. http://dx.doi.org/10.1504/ijwmc.2014.064815

[19] Bose, P., Morin, P., Stojmenovic, I. and Urrutia, J. (2001) Routing with Guaranteed Delivery in Ad Hoc Wireless Networks. Proceedings of the 3rd International Workshop on Discrete Algorithms and Methods for Mobile Computing and Communications, 609-616.

[20] Kuhn, F., Wattenhoffer, R. and Zollinger, A. (2003) Worst-Case Optimal and Average-Case Efficient Geometric AdHoc Routing. Proceedings of the 4th ACM International Symposium on Mobile Ad Hoc Networking \& Computing, 267-278. http://dx.doi.org/10.1145/778415.778447

[21] Stojmenovic, I. and Lin, X. (2001) Loop-Free Hybrid Single-Path/Flooding Routing Algorithms with Guaranteed Delivery for Wireless Networks. IEEE Transactions on Parallel and Distributed Systems, 12, 1023-1032. http://dx.doi.org/10.1109/71.963415

[22] Chen, D., Deng, J. and Varshney, P. (2007) Selection of a Forwarding Area for Contention-Based Geographic Forwarding in Wireless Multi-Hop Networks. IEEE Transactions on Vehicular Technology, 56, 3111-3122. http://dx.doi.org/10.1109/TVT.2007.900371

[23] Zhang, X.M., Wang, E.B., Xia, J.J. and Sung, D.K. (2013) A Neighbor Coverage-Based Probabilistic Rebroadcast for Reducing Routing Overhead in Mobile Ad Hoc Networks. IEEE Transactions on Mobile Computing, 12, 424-433. http://dx.doi.org/10.1109/tmc.2011.277

[24] Li, X.Y., Zhou, F. and Du, J.P. (2013) LDTS: A Lightweight and Dependable Trust System for Clustered Wireless Sensor Networks. IEEE Transactions on Information Forensics and Security, 8, 924-935. http://dx.doi.org/10.1109/TIFS.2013.2240299 